\title{
Pigmented Euglenophyceae of a lentic environment at the Upper Paraná River Floodplain, Brazil
}

\author{
Euglenophyceae pigmentadas de ambiênte lêntico da Planície de Inundação do \\ alto rio Paraná, Brasil
}

\section{Susicley Jati ${ }^{1 *}$, Carla Cristiane de Jesus Borsalli ${ }^{1}$ and Sueli Train ${ }^{2}$}

\author{
${ }^{1}$ Núcleo de Pesquisa em Limnologia, Ictiologia e Aquicultura - Nupélia, Universidade Estadual de \\ Maringá - UEM, Av. Colombo, 5790, Bloco H-90, CEP 87020-900, Maringá, PA, Brasil \\ ${ }^{2}$ Programa de Pós-graduação em Ecologia de Ambientes Aquáticos Continentais - PEA, \\ Universidade Estadual de Maringá, Av. Colombo, 5790, Bloco H-90, CEP 87020-900, Maringá, \\ PA, Brasil \\ *e-mail: susi@nupelia.uem.br
}

Cite as: Jati, S., Borsalli, C.C.J. and Train, S. Pigmented Euglenophyceae of a lentic environment at the Upper Paraná River Floodplain, Brazil. Acta Limnologica Brasiliensia, 2018, vol. 30, e212.

Abstract: Aim: Contributing to taxonomic knowledge on Pigmented Euglenophyceae at the Upper Paraná River Floodplain by enlarging the biodiversity survey in this ecosystem, in addition to contributing to the knowledge on the geographical distribution of this group in Brazil. Methods: Quarterly samplings in the limnetic region of a floodplain lake between February 2000 and March 2009, numbering 30 samples obtained from both vertical and horizontal trawls using plankton net of a $15 \mu \mathrm{m}$-mesh aperture and subjected to Transeau-solution preservation. We prepared temporary slides and analyzed in order to have the organisms identified, measured and photographed through digital camera, directly using an Olympus binocular optical microscope with a 400x/1000x magnification. The analysis included the calculation of Constancy (C) for the sample of each species. Results: 36 taxa were identified. Genus Trachelomonas Ehr. emend. Defl. had the best representation, with 20 taxa. Among the taxa surveyed, 53\% (19 taxa) constituted new records of occurrence for the Upper Paraná River Floodplain. The taxa recorded as frequent (33.3\%) are considered cosmopolitan and present wide geographical distribution throughout Brazil; however, most of the taxa surveyed (67\%) was classified as sporadic or rare, which is probably related to the high environmental variability observed along the study period. Conclusion: Among the surveyed 19 taxa constituted new records of occurrence for the upper Paraná River floodplain and extended the geographical distribution of Euglenophyceae in Brazil.

Keywords: taxonomy; floodplain lake; flagellated algae; Euglenophyceae.

Resumo: Objetivo: Contribuir para o conhecimento taxonômico das Euglenophyceae pigmentadas da planície de inundação do alto rio Paraná, ampliando o levantamento da biodiversidade neste ecossistema, além de contribuir para o conhecimento da distribuição geográfica do grupo no Brasil. Métodos: Foram realizadas amostragens trimestrais na região limnética de uma lagoa de várzea entre fevereiro de 2000 e março de 2009, totalizando 30 amostras. Estas foram obtidas a partir de arrastos verticais e horizontais com rede de plâncton com $15 \mu \mathrm{m}$ de abertura de malha e preservadas em soluçâo de Transeau. Foram preparadas e analisadas lâminas temporárias, nas quais os organismos foram identificados, medidos e fotografados em câmara digital, diretamente no microscópio óptico binocular Olympus em aumento de 400x e 1000x. Foi calculada a constância (C) de cada espécie nas amostras analisadas. Resultados: Foram identificados 36 táxons. O gênero Trachelomonas Ehr. emend. Defl. foi 
o melhor representado com 20 táxons. Dentre os táxons inventariados 53\% (19 táxons) constituem novos registros de ocorrência para a Planície de inundação do alto rio Paraná. Os táxons registrados como frequentes $(33,3 \%)$ são considerados cosmopolitas e apresentam ampla distribuição geográfica no Brasil, no entanto, a maioria dos táxons inventariados (67\%) foi classificada como esporádica ou rara, o que provavelmente está relacionado à grande variabilidade ambiental observada durante o período de estudo. Conclusáo: Entre os táxons descritos, 19 táxons constituíram novos registros de ocorrência para a Planície de Inundação do Alto Rio Paraná e ampliam a distribuição geográfica das Euglenophyceae para o Brasil.

Palavras-chave: taxonomia; lagoa de várzea; alga flagelada; Euglenofíceas.

\section{Introduction}

Floodplains are river systems with high heterogeneity of habitats and great functional and structural complexity (Thomaz et al., 2004). In these systems, the hydro sedimentological regime (Neiff, 1990) acts directly on the degree of connectivity among their many environments, providing an efficient route of dispersion and recruitment of species as well as ensuring the maintenance of high biodiversity (Train \& Rodrigues, 2004; Borges \& Train, 2009). The Upper Paraná River Floodplain is considered a relevant region for the conservation of biodiversity in Brazil and its environments have been studied since 2000 through a Long-term Ecological Project (PELD-site 6/ CNPq), which has constituted an important opportunity to build a survey of phytoplankton in this environment.

Phytoplanktonic communities of river-floodplain systems present high diversity of species distributed in many taxonomic algae classes, including the Euglenophyceae, a group especially favored in floodplain lakes rich in organic matter and with the occurrence of high species richness (Jati \& Train, 1994; Train \& Rodrigues, 2004).

Even though the ecology of the phytoplanktonic community in these environments has been widely investigated, studies involving its taxonomic approach are still rare (Jati \& Train, 1994; Oliveira et al., 1994; Bovo-Scomparin et al., 2005, Paula et al., 2014; Jati et al., 2014; Moresco et al., 2015). In this context, the papers by Jati \& Train (1993, 1994) and Biolo \& Rodrigues (2010) represent the only records of Euglenophyceae taxonomy for such environments.

Therefore, the objective of this study is to contribute to the taxonomic knowledge on Pigmented Euglenophyceae at the Upper Paraná River Floodplain as well as to expand the biodiversity survey in this ecosystem, in addition to contributing to the knowledge on the geographical distribution of this group in Brazil.

\section{Material and Methods}

The Upper Paraná River Floodplain is located in the Central-South region of Brazil, encompassing the area between Porto Primavera (Primavera/SP) and Itaipu hydroelectric plants (Guaíra/PR). With $230 \mathrm{~km}$ of extension, it is the last dam-free stretch of Paraná River, whose major channel presents numerous islands, marginal lakes and channels (Souza Filho, 2009).

This study was developed in a floodplain lake (Pau Véio, 22 $44^{\prime}$ S; 531' $\mathrm{W}$ ) permanently connected to the Paraná River, located at Mutum Island (Figure 1) and presenting approximately $1.2 \mathrm{~km}$ of length and $50 \mathrm{~m}$ of width; with an area of 3.0 ha and an average depth of $1.8 \mathrm{~m}$. The lake is surrounded by arboreous vegetation and its margins are occupied with aquatic macrophytes, especially Eichhornia azurea Kunth.

We carried out quarterly samplings at the limnetic region of the lake between February 2000 and March 2009, totaling 30 samples obtained from vertical and horizontal trawls using plankton net with a $15-\mu \mathrm{m}$-mesh aperture and subjected to Transeau solution preservation (Bicudo \& Menezes, 2006).

We prepared and analyzed temporary slides until new taxa stopped occurring. The organisms were identified, measured and photographed through digital camera directly using an Olympus binocular optical microscope with a 400x/1000x magnification. The taxonomic framework followed Bicudo \& Menezes (2016). Species and infraspecific taxa were identified according to basic works such as Deflandre (1926), Huber-Pestalozzi (1955), and Tell \& Conforti (1986). Several recent floristic works as Alves-da-Silva \& Klein (2015), Alves-da-Silva et al. (2013, 2016), Araujo \& Bicudo (2017), Araujo et al. (2012), Kufner \& Giani (2017) were also used, in addition to molecular biology studies such as Bennett \& Triemer (2012), Kim et al. (2013) and Marin et al. (2003).

In order to estimate the constancy (C) of each species in the samples analyzed, we used the suggestion by Dajoz (2005): $\mathrm{C}=(\mathrm{p} \times 100) / \mathrm{P}$, 


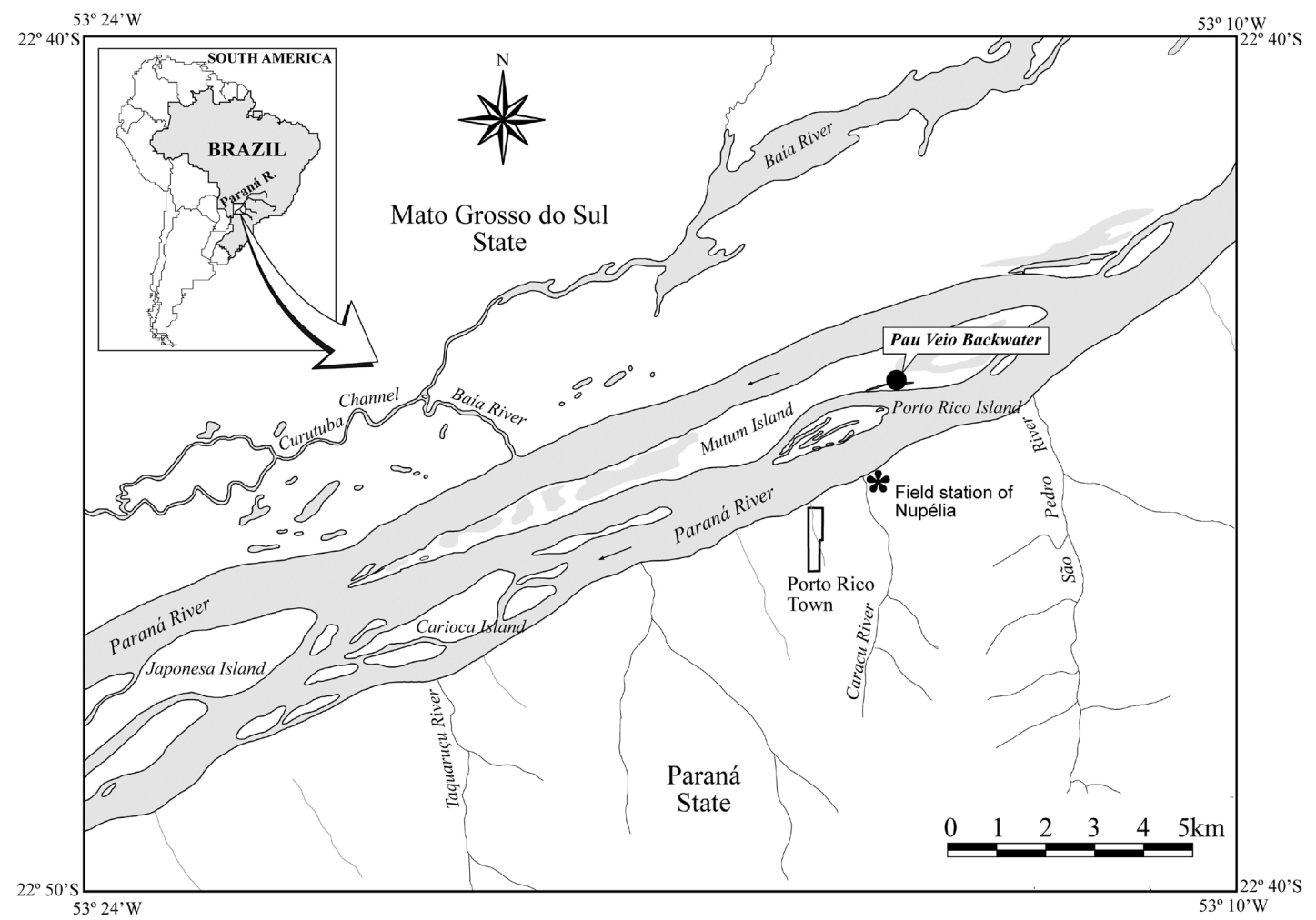

Figure 1. Map and location da sampling station.

according to which: $\mathrm{C} \geq 70$ represents the constant species; $70 \geq C \geq 30$ the frequent species; $30 \geq C \geq 10$ the sporadic species, and $\mathrm{C}<10$ represents the rare species. The character " $\mathrm{p}$ " is the amount of samples containing the species and " $\mathrm{P}$ " is the total amount of samples analyzed.

The samples are stored at the Herbário da Universidade Estadual de Maringá (HUEM Herbarium of the State University of Maringá).

Concomitantly to phytoplankton samplings, we also sampled physical and chemical water parameters to verify the variation of the environmental conditions along the study period. Water temperature (WT, ${ }^{\circ} \mathrm{C}$ ), $\mathrm{pH}$, electrical conductivity (Cond, $\mu \mathrm{Scm}^{-1}$ ), and dissolved oxygen (DO, $\mathrm{mgL}^{-1}$ ) were obtained in situ using Digimed portable digital potentiometers. Water transparency $(\mathrm{m})$ was obtained using a Secchi disk. Concentrations of total phosphorus (TP, $\mu \mathrm{gL}^{-1}$ ), reactive soluble phosphorus ( $\mathrm{PO}_{4} \mu \mathrm{gL}^{-1}$; Golterman et al., 1978), total nitrogen Kjeldahl (TN, $\mu \mathrm{gL}^{-1}$; Mackereth et al.,1978) nitrate ( $\mathrm{NO}_{3}-\mathrm{N}, \mu \mathrm{gL}^{-1}$; Giné et al., 1980), and ammonium $\left(\mathrm{NH}_{4}-\mathrm{N}, \mu \mathrm{gL}^{-1}\right.$; Koroleff, 1978) were also obtained.

\section{Results and Discussion}

The environmental variables sampled simultaneously with the phytoplankton samples evidence high environmental variability during the study period. Table 1 presents the temporal variation of the mean values of the main environmental variables along with their respective standard deviations. The environmental variation caused by the hydrometric pulse seasonality and the range of variation of this event between the sampling years (Figure 2) are probably the main factors responsible for the high variability observed for the period. Such a high environmental variability may have benefited the great amount of both rare and sporadic species surveyed as well as the fact that the species classified as frequent are pointed out as cosmopolitan in the literature (Alves-da-Silva \& Bridi, 2004).

The taxonomic framework considered in this study followed Bicudo \& Menezes (2016).

Division Euglenophyta

Class Euglenophyceae

Ordem Euglenales

Family Phacaceae Kim et al. 2010

Genus Lepocinclis Perty emend. Marin \& Melkonian 2003

Phacus Dujardin emend. Linton \& Karnkowska 2010

Family Euglenaceae Stein 1878 
Genus Monomorphina Mereschkowsky 1877 emend. Kosmala \& Zakryś 2007

Strombomonas Deflandre 1930

Trachelomonas Ehrenberg 1835

We identified 36 taxa belonging to the families Phacaceae (12) and Euglenaceae (24) (Figure 3-38). The genus Trachelomonas had the best representation, with twenty (20) taxa, followed by Lepocinclis and Phacus, both with six (6) taxa, Strombomonas with three (3) and Monomorphina with one (1) taxon.

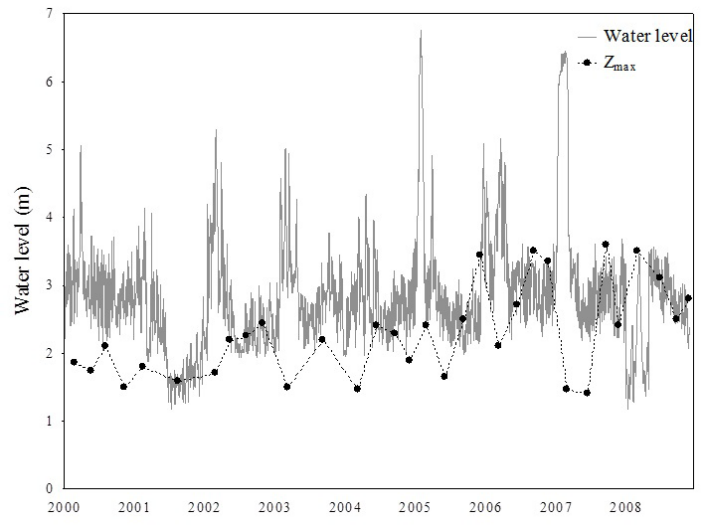

Figure 2. Hydrometric levels of the Paraná River (lines) and Zmax of the floodplain lake (points) from February 2000 to March 2009. Points also indicate the sampling days.
Among the surveyed taxa, 55.5\% (19 taxa) constituted new records of occurrence for the Upper Paraná River Floodplain, which are the following: Lepocinclis fusca, Monomorphina pyrum, Phacus contortus, P. longicauda var. major, P. rodriguesiae, Strombomonas fluviatilis var. levis, S. triquetra, Trachelomonas abrupta var. obesa, T. acanthophora, T. amphoriformis var. spinosa, T. armata var. longispina, T. decora, T. hispida var. coronata, T. megalacantha, T. molesta, T. obtusa, T. similis var. similis, T. volvocina var. volvocina and T. woycickii var. pusilla.

Frequent taxa included: Lepocinclis acus var. acus $(45 \%)$, L. oxyuris var. oxyuris (41\%), L. ovum var. ovum (42\%), L. salina var. salina (45\%), L. spinosa (54\%), Phacus orbicularis (45\%), P. longicauda var. major (33\%), Trachelomonas armata var steini (42\%), T. hispida var. crenullatocollis (33\%), T. rugulosa var. rugulosa fa. paralela (30\%), T. sculpta var. sculpta (51\%), and T. volvocinopsis var. volvocinopsis (67\%). Most of the taxa surveyed (67\%) was classified as sporadic or rare.

Lepocinclis acus (O.F. Müller) Marin \& Melkonian var. acus, Protist 154: 104. 2003: 104.

Figure 3

Fusiform cells; long and thin caudal process; helicoidal striation hardly discernible; rod-shaped paramylon granules; numerous peripheral discoid

Table 1. Mean values and coefficient of variation (\% in parentheses) of the abiotic variables sampled in the Pau Véio lake between March 2000 and March 2009.

\begin{tabular}{cccccccccccccc}
\hline & Depth & $\mathbf{T}$ & $\mathbf{D O}$ & $\mathbf{p H}$ & Cond. & Secchi & Turb. & Alc. & $\mathbf{T N}$ & $\mathbf{N O}_{3}-\mathbf{N}$ & $\mathbf{N H}_{4}-\mathbf{N}$ & $\mathbf{T P}^{2}$ & $\mathbf{P O}_{4}-\mathbf{P}$ \\
\hline 2000 & 1.8 & 24.6 & 6.1 & 6.6 & 52.8 & 1.0 & 7.7 & 314.3 & 273.9 & 44 & 6.2 & 15 & 0.7 \\
& $(71.7)$ & $(1.0)$ & $(77.5)$ & $(23.5)$ & $(0.5)$ & $(463.9)$ & $(4.2)$ & $(0.6)$ & $(0.4)$ & $(146.0)$ & $(852.2)$ & $(40.5)$ & $(713.8)$ \\
2001 & 1.7 & 27 & 6.9 & 6.62 & 54.0 & 1.1 & 53.5 & 270.4 & 464.4 & 146.3 & 10.45 & 19.6 & 4.8 \\
& $(8.3)$ & $(18.8)$ & $(8.4)$ & $(1.9)$ & $(8.5)$ & $(0)$ & $(127.8)$ & $(32.5)$ & $(32.1)$ & $(16.2)$ & $(106.2)$ & $(33.1)$ & $(58.9)$ \\
2002 & 2.1 & 25.1 & 5.3 & 6.5 & 58.0 & 1.8 & 2.2 & 351 & 193.3 & 62.9 & 17.7 & 16.2 & 3.5 \\
& $(14.8)$ & $(14.8)$ & $(42.4)$ & $(5.6)$ & $(14.5)$ & $(23.1)$ & $(13.2)$ & $(47.3)$ & $(19.6)$ & $(67.5)$ & $(118.9)$ & $(11.9)$ & $(14.5)$ \\
2003 & 1.8 & 25.8 & 5.73 & 6.6 & 61.4 & 1.4 & 1.7 & 322.5 & 224.1 & 36.7 & 4.7 & 36.0 & 7.7 \\
& $(26.7)$ & $(30.1)$ & $(16.2)$ & $(4.4)$ & $(13.1)$ & $(69.5)$ & $(103.9)$ & $(0.6)$ & $(12.9)$ & $(25.9)$ & $(38.1)$ & $(50.1)$ & $(94.0)$ \\
2004 & 2.0 & 25.1 & 4.7 & 6.2 & 56.7 & 2.0 & 3.5 & 344.4 & 197.4 & 68.4 & 18.0 & 14.3 & 3.1 \\
& $(21.5)$ & $(20.4)$ & $(41.4)$ & $(4.9)$ & $(9.7)$ & $(21.5)$ & $(86.3)$ & $(11.0)$ & $(26.8)$ & $(93.8)$ & $(13.7)$ & $(49.3)$ & $(65.2)$ \\
2005 & 2.5 & 24.1 & 4.2 & 6.3 & 58.1 & 2.1 & 4.3 & 399 & 484.4 & 75.3 & 11.8 & 16.8 & 3.4 \\
& $(29.5)$ & $(16.0)$ & $(33.4)$ & $(3.5)$ & $(6.8)$ & $(53.6)$ & $(89.0)$ & $(12.8)$ & $(54.6)$ & $(65.2)$ & $(36.9)$ & $(62.7)$ & $(40.3)$ \\
2006 & 2.9 & 24.8 & 5.3 & 6.4 & 55.1 & 2.9 & 3.2 & 351.3 & 398.3 & 59.9 & 5.7 & 13.7 & 6.8 \\
& $(22.0)$ & $(14.9)$ & $(27.5)$ & $(7.0)$ & $(10.3)$ & $(22.0)$ & $(101.7)$ & $(8.9)$ & $(96.2)$ & $(41.7)$ & $(80.5)$ & $(46.7)$ & $(60.9)$ \\
2007 & 2.2 & 25.4 & 5.4 & 6.8 & 56.5 & 2.0 & 6.5 & 414.2 & 381.1 & 97.6 & 16.2 & 17.7 & 7.4 \\
& $(46.6)$ & $(14.7)$ & $(47.8)$ & $(6.7)$ & $(7.8)$ & $(39.0)$ & $(124.8)$ & $(9.5)$ & $(18.1)$ & $(71.8)$ & $(85.3)$ & $(24.7)$ & $(83.3)$ \\
2008 & 2.9 & 23.6 & 4.1 & 6.6 & 58.5 & 2.3 & 2.7 & 420.9 & 409.0 & 88.3 & 16.9 & 16 & 7.8 \\
& $(14.3)$ & $(17.0)$ & $(37.4)$ & $(1.8)$ & $(11.5)$ & $(20.4)$ & $(82.0)$ & $(11.6)$ & $(15.8)$ & $(43.1)$ & $(104.3)$ & $(26.8)$ & $(58.2)$ \\
$2009 *$ & 2.8 & 28.5 & 5.22 & 58.8 & 2.25 & 0.96 & 2.8 & 410.4 & 999.0 & 120.6 & 7.2 & 20.6 & 5.5 \\
\hline
\end{tabular}

Depth (m); T, Water temperature $\left({ }^{\circ} \mathrm{C}\right)$; DO, dissolved oxygen ( $\left.\mathrm{mg} \mathrm{L}-1\right)$; Cond., electrical conductivity $\left(\mu \mathrm{cm}^{-1}\right)$; Secchi disk (m); Turb., Turbidity (NTU); Alc., Alkalinity $\left(\mathrm{mg} \mathrm{L}^{-1}\right)$; Clo, Chlorophyl-a ( $\left.\mu \mathrm{g} \mathrm{L}^{-1}\right)$; TN, Total nitrogen ( $\left.\mu \mathrm{g} \mathrm{L} \mathrm{L}^{-1}\right)$; $\mathrm{NO}_{3}$, Nitrate $\left(\mu \mathrm{g} \mathrm{L}^{-1}\right) ; \mathrm{NH}_{4}$, Ammonium $\left(\mu \mathrm{g} \mathrm{L}^{-1}\right)$; TP, total phosphorus $\left(\mu \mathrm{g} \mathrm{L}^{-1}\right) ; \mathrm{PO}_{4}$, Phosphate $\left(\mu \mathrm{g} \mathrm{L}{ }^{-1}\right) ;{ }^{*}$ Data referent to only one sampling in the year of 2009 . 

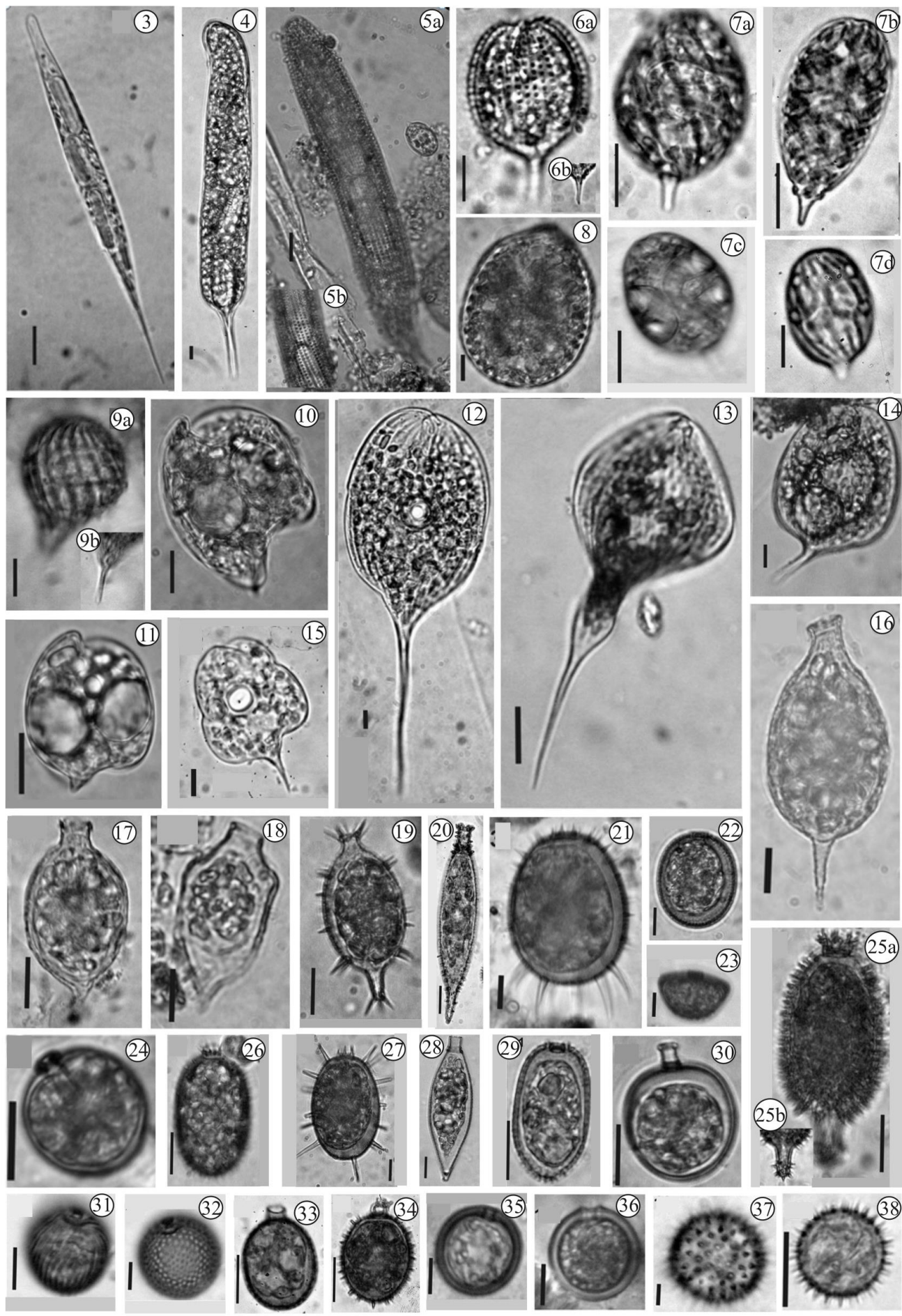

Figures 3-38. 3. Lepocinclis acus var. acus, 4. L. oxyuris var. oxyuris, 5a. L. fusca, 5b. Detail pellicular strips, 6a. Lepocinclis spinosa, 6b. Detail of the caudal process, 7 a-d. L. ovum var. ovum, 8. L. salina var. salina, 9a. Monomorphina pyrum, 9b. Detail of the caudal process, 10. Phacus contortus var. contortus, 11. P. curvicauda var. curvicauda, 12. P. longicauda var. major, 13. P. tortus, 14. P. orbicularis, 15. P. rodriguesiae var. rodriguesiae, 16. Strombomonas fluviatilis var. levis, 17. S. fluviatilis var. fluviatilis, 18. S. triquetra var. torta, 19. Trachelomonas acanthophora var. acanthophora, 20. T. amphoriformis var. spinosa, 21. T. armata var. longispina, 22. T. abrupta var. obesa, 23. T. cupula, 24. T. cervicula var. cervicula, 25a. T. decora var. decora, 25b. Detail of the caudal process, 26. T. hispida var. coronata, 27. T. megalacantha var. megalacantha, 28. T. molesta var. molesta, 29. T. obtusa var. obtusa, 30. T. parvicollis var. parvicollis, 31. T. rugulosa var. rugulosa fa. paralela, 32. T. sculpta var. sculpta, 33. T. similis var. similis, 34. T. similis var. spinosa, 35. T. volvocina var. volvocina, 36. T. volvocinopsis var. volvocinopsis, 37. T. woycickii var. woycickii, 38. T. woycickii var. pusilla. Scale bar $=10 \mu \mathrm{m}$. 
chloroplasts, several long. Length $97-170 \mu \mathrm{m}$, width 7.3-15.6 $\mu \mathrm{m}$.

Occurrence frequency: Frequent.

Examined material: Brazil, Paraná, Porto Rico, 09.II.01, L. C. Rodrigues w/n $n^{O}$ (HUEM 16753); 03.VIII.02, L. C. Rodrigues $w / n^{O}$ (HUEM 16758); 02.XI.02, L. C. Rodrigues $w / n^{O}$ (HUEM 16759); 08.III.03, L. C. Rodrigues w/no (HUEM 16760); 02.XII.04, L. C. Rodrigues $w / n^{O}$ (HUEM 16762); 08.III.04, L. C. Rodrigues w/no (HUEM 16763); 24.IX.04, L. C. Rodrigues $w / n^{O}$ (HUEM 16765); 03.III.05, S. Jati w/no (HUEM 16766); 09.XII.05, S. Jati $w / n^{O}$ (HUEM 16769); 10.III.06, S. Jati $w / n^{O}$ (HUEM 16770); 23.VI.06, S. Jati w/no (HUEM 16771); 01.XII.06, S. Jati w/no (HUEM 16772); 29.II.08, S. Jati w/no (HUEM 16778); 27.XI.08, S. Jati $w / n^{O}$ (HUEM 16779); 13.III.09, S. Jati $w / n^{O}$ (HUEM 16782).

Lepocinclis oxyuris Schmarda Marin \& Melkonian var. oxyuris, Protist 154: 104. 2003.

Figure 4

Cylindrical cell; circular apical view; striated periplast in spiral; twisted anterior pole; posterior pole with tapered long caudal process; two bastoniform paramylons, one anterior and another posterior to the core; several discoid chloroplasts all over the cell. Length 133-254 $\mu \mathrm{m}$, width 27-36.3 $\mu \mathrm{m}$, caudal process $26.1 \mu \mathrm{m}$, paramylons length $39.6 \mu \mathrm{m}$, width $13.2 \mu \mathrm{m}$.

Occurrence frequency: Frequent.

Examined Material: Brazil, Paraná, Porto Rico, 11.V.00, L. C. Rodrigues $w / n^{O}$ (HUEM 16754); 20.VIII.01, L. C. Rodrigues $w / n^{O}$ (HUEM 16755); 03.VIII.02, L. C. Rodrigues $w / n^{O}$ (HUEM 16758); 02.XI.02, L. C. Rodrigues w/no (HUEM 16759); 08.III.03, L. C. Rodrigues w/no (HUEM 16760); 02.XII.04, L. C. Rodrigues $w / n^{O}$ (HUEM 16762); 08.III.04, L. C. Rodrigues w/no (HUEM 16763); 24.IX.04, L. C. Rodrigues w/no (HUEM 16765); 03.III.05, S. Jati w/no (HUEM 16766); 23.VI.06, S. Jati $w / n^{O}$ (HUEM 16771); 01.XII.06, S. Jati $w / n^{O}$ (HUEM 16772); 15.IX.06, S. Jati $w / n^{O}$ (HUEM 16773); 29.II.08, S. Jati w/no (HUEM 16778); 13.III.09, S. Jati w/no (HUEM 16782).

Lepocinclis fusca (Klebs) Kosmala \& Zakryś, Journal of Phycology 41: 1264. 2005.

Figure $5 \mathrm{a}$ and $5 \mathrm{~b}$

Cylindrical cell; rounded anterior and posterior pole with long caudal process; striated periplast in spiral in which polygonal warts are arranged on the striae; two blastoniform paramylon granules, one anterior and another posterior to the core, several discoid chloroplasts. Length 137.8-190.5 $\mu \mathrm{m}$, width 23.5-24.6 $\mu \mathrm{m}$, caudal process of $26.1 \mu \mathrm{m}$.

Comment: The observed specimens constitute a new record for the Upper Paraná River Floodplain.

Occurrence frequency: Sporadic.

Examined Material: Brazil, Paraná, Porto Rico, 09.II.01, L. C. Rodrigues $w / n^{O}$ (HUEM 16753); 03.III.05, S. Jati w/no (HUEM 16766); 01.XII.06, S. Jati $w / n^{O}$ (HUEM 16772); 27.XI.08, S. Jati $w / n^{O}$ (HUEM 16779).

Lepocinclis ovum (Ehrenberg) Minkevich var. ovum, Trudy Imperatorskago S. Peterburgskavo Obshchestra Estestvoispytatelei. Vypusk 29: 241. 1899.

Figure $7 \mathrm{a}, 7 \mathrm{~b}, 7 \mathrm{c}$ and $7 \mathrm{~d}$

Ovoid organisms; levogyre striae; rounded anterior pole and posterior pole ending in a conical caudal process; two lateral, horseshoe-shaped paramylon granules; several discoid chloroplasts. Length 28.7-31.3 $\mu \mathrm{m}$, width 15.6-23.5 $\mu \mathrm{m}$., length of caudal process 5.2-9.9 $\mu \mathrm{m}$.

Comment: According to Tell \& Conforti (1986), the thickening of the caudal process is a characteristic that distinguishes the typical variety of Lepocinclis ovum var. bütschlii; however, according to Menezes (1987), within a single population such feature represents a morphological expression of the cellular development. The observed specimens presented high morphological variation, such as observed by Jati \& Train (1994) for the examined material at Porto Rico Island.

Occurrence frequency: Frequent.

Examined Material: Brazil, Paraná, Porto Rico, 11.V.00, L. C. Rodrigues $w / n^{O}$ (HUEM 16754); 20.VIII.01, L. C. Rodrigues $w / n^{O}$ (HUEM 16755); 03.VIII.02, L. C. Rodrigues $w / n^{O}$ (HUEM 16758); 02.XI.02, L. C. Rodrigues w/no (HUEM 16759); 08.III.03, L. C. Rodrigues w/no (HUEM 16760); 02.XII.04, L. C. Rodrigues $w / n^{O}$ (HUEM 16762); 08.III.04, L. C. Rodrigues w/n ${ }^{\circ}$ (HUEM 16763); 24.IX.04, L. C. Rodrigues w/no (HUEM 16765); 03.III.05, S. Jati w/n ${ }^{O}$ (HUEM 16766); 16.IX.05, S. Jati $w / n^{O}$ (HUEM 16768); 09.XII.05, S. Jati $w / n^{O}$ (HUEM 16769); 10.III.06, S. Jati $w / n^{O}$ (HUEM 16770); 15.IX.06, S. Jati w/no (HUEM 16773); 27.XI.08, S. Jati $w / n^{O}$ (HUEM 16779); 13.III.09, S. Jati $w / n^{O}$ (HUEM 16782).

Lepocinclis salina Fritsch var. salina, New Phytologist. 13: 351 fig.3 a, b, e. 1940

Figure 8

Ovoid cell; circular apical view; dextrogyre striae; rigid periplast; neatly asymmetric anterior pole with subapical flagella aperture; rounded 
posterior pole; large grain, discoid or elliptical, never bastoniform paramylon granules; several discoid chloroplasts. Length 33.9-54.1 $\mu \mathrm{m}$, width 26.1-49.2 $\mu \mathrm{m}$.

Occurrence frequency: Frequent.

Examined Material: Brazil, Paraná, Porto Rico, 11.V.00, L. C. Rodrigues $w / n^{O}$ (HUEM 16754); 09.II.01, L. C. Rodrigues w/no (HUEM 16753); 20.VIII.01, L. C. Rodrigues $w / n^{O}$ (HUEM 16755); 03.VIII.02, L. C. Rodrigues $w / n^{O}$ (HUEM 16758); 03.IX.03, L. C. Rodrigues w/no (HUEM 16761); 02.XII.04, L. C. Rodrigues $w / n^{O}$ (HUEM 16762); 08.III.04, L. C. Rodrigues w/no (HUEM 16763); 24.IX.04, L. C. Rodrigues w/no (HUEM 16765); 03.III.05, S. Jati w/no (HUEM 16766); 10.VI.05, S. Jati $w / n^{O}$ (HUEM 16767); 16.IX.05, S. Jati $w / n^{O}$ (HUEM 16768); 10.III.06, S. Jati w/no (HUEM 16770); 23.VI.06, S. Jati w/no (HUEM 16771); 27.XI.08, S. Jati $w / n^{\circ}$ (HUEM 16779); 13.III.09, S. Jati $w / n^{O}$ (HUEM 16782).

Lepocinclis spinosa Bennett \& Triemer, Journal of Phycology 48(3): 837. 2012.

Figure $6 a$ and $6 b$

Napiform cells, spines curved on the striae towards the posterior pole; anterior pole with median papilla emerging; conical caudal process; numerous discoid chloroplasts. Length 29.7-39.6 $\mu \mathrm{m}$, width 26.4-36.3 $\mu \mathrm{m}$, length caudal process. 6.6-10,0 $\mu \mathrm{m}$.

Occurrence frequency: Frequent.

Examined Material: Brazil, Paraná, Porto Rico, 11.V.00, L. C. Rodrigues $w / n^{O}$ (HUEM 16754); 20.VIII.01, L. C. Rodrigues $w / n^{O}$ (HUEM 16755); 28.II.02, L. C. Rodrigues $w / n^{O}$ (HUEM 16756); 03.VIII.02, L. C. Rodrigues $w / n^{O}$ (HUEM 16758); 08.III.03, L. C. Rodrigues w/no (HUEM 16760); 02.XII.04, L. C. Rodrigues $w / n^{O}$ (HUEM 16762); 08.III.04, L. C. Rodrigues w/no (HUEM 16763); 18.VI.04, L. C. Rodrigues $w / n^{O}$ (HUEM 16764); 24.IX.04, L. C. Rodrigues $w / n^{O}$ (HUEM 16765); 03.III.05, S. Jati w/no (HUEM 16766); 10.VI.05, S. Jati $w / n^{O}$ (HUEM 16767); 09.XII.05, S. Jati $w / n^{O}$ (HUEM 16769); 10.III.06, S. Jati $w / n^{O}$ (HUEM 16770); 23.VI.06, S. Jati w/no (HUEM 16771); 15.IX.06, S. Jati w/no (HUEM 16773); 29.II.08, S. Jati w/no (HUEM 16778); 27.XI.08, S. Jati $w / n^{\circ}$ (HUEM 16779); 13.III.09, S. Jati $w / n^{O}$ (HUEM 16782).

Monomorphina pyrum (Ehrenberg) Mereschkowsky emend Kim, Triemer \& Shin, Journal of Phycology 49:88. 2013.

Figure $9 \mathrm{a}$ and $9 \mathrm{~b}$

Obpyriform cell; levogyre striae forming conspicuous hyaline keels; rounded anterior pole; posterior pole ending in a pointed caudal process; two flat ring-shaped paramylon granules located between the pellicle and the single perforated parietal chloroplast, looking like several smaller discoid chloroplasts. Length $52.2 \mu \mathrm{m}$, width $32.5 \mu \mathrm{m}$.

Comment: The observed specimens constitute a new record for the Upper Paraná River Floodplain.

Occurrence frequency: Rare.

Examined Material: Brazil, Paraná, Porto Rico, 01.XII.06, S. Jati w/n ${ }^{O}$ (HUEM 16772); 13.III.09, S. Jati $w / n^{O}$ (HUEM 16782).

Phacus contortus Bourrelly var. contortus, Algues d'eau douce de la Guadeloupe et dependances 177, pl. 22, fig. 271-277 1952.

Figure 10

Ovoid cells, composed of two asymmetrical and twisted alliform parts, separated between each other by large, deep grooves, cuneiform apical view, short and curved caudal process; two discoid paramylon granules arranged side by side, other smaller, bastoniform dispersed by the cytoplasm, numerous discoid chloroplasts. Length 40.8-44.3 $\mu \mathrm{m}$, width 32.6-34.4 $\mu \mathrm{m}$.

Comment: The observed specimens constitute a new record for the Upper Paraná River Floodplain.

Occurrence frequency: Rare.

Examined Material: Brazil, Paraná, Porto Rico, 03.III.05, S. Jati w/n ${ }^{\circ}$ (HUEM 16766); 29.II.08, S. Jati $w / n^{O}$ (HUEM 16778).

Phacus curvicauda Swirenko var. curvicauda, Archiv für Hydrobiologie und Planktonkunde 10: 333, pl. 2, fig. 13-16. 1915.

\section{Figure 11}

Ovoid cells; sigmoid apical view; short and curved caudal process; two discoid paramylon granules in the arranged side by side, other smaller discoid or bastoniform ones. Length 19.7-29.6 $\mu \mathrm{m}$, width 17.2-24.5 $\mu \mathrm{m}$, length caudal process $5 \mu \mathrm{m}$.

Occurrence frequency: Sporadic.

Examined Material: Brazil, Paraná, Porto Rico, 03.III.05, S. Jati $w / n^{O}$ (HUEM 16766); 16.IX.05, S. Jati $w / n^{O}$ (HUEM 16768); 10.III.06, S. Jati $w / n^{O}$ (HUEM 16770); 23.VI.06, S. Jati w/n ${ }^{\circ}$ (HUEM 16771); 15.IX.06, S. Jati w/no (HUEM 16773); 29.II.08, S. Jati $w / n^{\circ}$ (HUEM 16778); 27.XI.08, S. Jati $w / n^{O}$ (HUEM 16779).

Phacus longicauda (Ehrenberg) Dujardin var. major (Ehrenberg) Dujardin, Histoire Naturelle des Zoophytes, 337, pl.5, fig. 6. 1841.

Figure 12

Obovoid cells, long, straight caudal process; two concentric and central discs of paramylon, 
numerous discoid chloroplasts. Length $198 \mu \mathrm{m}$, width $70-75 \mu \mathrm{m}$, length caudal process $39.4 \mu \mathrm{m}$.

Comment: The observed specimens constitute a new record for the Upper Paraná River Floodplain.

Occurrence frequency: Frequent.

Examined Material: Brazil, Paraná, Porto Rico, 02.XI.02, L. C. Rodrigues $w / n^{O}$ (HUEM 16759); 08.III.03, L. C. Rodrigues w/no (HUEM 16760); 02.XII.04, L. C. Rodrigues $w / n^{O}$ (HUEM 16762); 24.IX.04, L. C. Rodrigues $w / n^{O}$ (HUEM 16765); 03.III.05, S. Jati w/no (HUEM 16766); 09.XII.05, S. Jati $w / n^{O}$ (HUEM 16769); 23.VI.06, S. Jati $w / n^{O}$ (HUEM 16771); 15.IX.06, S. Jati w/no (HUEM 16773); 29.II.08, S. Jati w/n (HUEM 16778); 27.XI.08, S. Jati w/no (HUEM 16779); 13.III.09, S. Jati $w / n^{O}$ (HUEM 16782).

Phacus orbicularis Hübner emend Zakryś \& Kosmala, Journal of Phycology 43: 1077, fig.1, f-n, p, r, s, u. 2007.

Figure 14

Cell broadly ovoid to circular; triangular apical view; periplast with transverse striae between longitudinal striae; long and curved caudal process; two central, overlapping paramylon discs of different sizes, numerous discoid chloroplasts. Length 39.8-83.5 $\mu \mathrm{m}$, width 27.5-59.4 $\mu \mathrm{m}$, length caudal process $13.2-23.1 \mu \mathrm{m}$.

Occurrence frequency: Frequent.

Examined Material: Brazil, Paraná, Porto Rico, 11.V.00, L. C. Rodrigues $w / n^{O}$ (HUEM 16754); 09.II.01, L. C. Rodrigues $w / n^{O}$ (HUEM 16753); 20.VIII.01, L. C. Rodrigues $w / n^{O}$ (HUEM 16755); 03.VIII.02, L. C. Rodrigues $w / n^{O}$ (HUEM 16758); 02.XI.02, L. C. Rodrigues $w / n^{\circ}$ (HUEM 16759); 08.III.03, L. C. Rodrigues w/no (HUEM 16760); 02.XII.04, L. C. Rodrigues $w / n^{O}$ (HUEM 16762); 08.III.04, L. C. Rodrigues w/no (HUEM 16763); 24.IX.04, L. C. Rodrigues w/no (HUEM 16765); 03.III.05, S. Jati w/no (HUEM 16766); 10.III.06, S. Jati $w / n^{O}$ (HUEM 16770); 23.VI.06, S. Jati w/n ${ }^{O}$ (HUEM 16771); 01.XII.06, S. Jati w/n ${ }^{O}$ (HUEM 16772); 27.XI.08, S. Jati w/n ${ }^{\circ}$ (HUEM 16779); 13.III.09, S. Jati w/no (HUEM 16782).

Phacus rodriguesiae Conforti var. rodriguesiae, Revue Hydrobiologique Tropicale, v.27, p.15, pl.IV, figs. 5 a-c, 1994.

Figure 15

Ovoid cell; rounded anterior pole; marginal notches located in the middle of the cell; median groove encompassing 1/3 of the length; hyaline, straight, conical caudal process; two paramylon discs, a larger central one and another smaller, lateral one. Length 23.5-36.9 $\mu \mathrm{m}$, width 21.4-24.6 $\mu \mathrm{m}$, length caudal process 7.4-8.2 $\mu \mathrm{m}$.

Comment: This taxon is rarely mentioned in the specialized Brazilian literature; it has been mentioned by Alves-da-Silva et al. (1991) for Rio Grande do Sul as Phacus acuminatus Stokes var. acuticauda Pochm. The observed specimens constitute a new record for the Upper Paraná River Floodplain.

Occurrence frequency: Rare.

Examined Material: Brazil, Paraná, Porto Rico, 13.III.09, S. Jati $w / n^{O}$ (HUEM 16782).

Phacus tortus (Lemmermann) Skvortzov, Berichte der Deutschen Botanischen Gesellschaft, 46(2):110, pl.2, fig. 9-10. 1928.

Figure 13

Ovoid cell; median groove encompassing $1 / 3$ of the length; rounded anterior pole; long, twist on the caudal process; two concentric and central discs of paramylon, numerous discoid chloroplasts. Length 67.7-198 $\mu \mathrm{m}$, width 31.3-70.5 $\mu \mathrm{m}$, caudal process 39.4-97.5 $\mu \mathrm{m}$.

Comment: Tell \& Conforti (1986) considered the degree of cell torsion as a basic characteristic for the differentiation of Phacus tortus (one turn of torsion), Phacus sesquitortus (one and $1 / 2$ turn of torsion) and Phacus helicoides (many turns of torsion). However, Alves-da-Silva \& Bicudo (2009) consider this a not very consistent morphological feature. In the same way, Alves-da Silva \& Klein (2015) raised to species the taxa similar to Phacus longicauda (Ehrenberg) Dujardin var. tortus Lemmerman. We agree with this last author and identify our specimens as $P$. tortus.

Occurrence frequency: Sporadic.

Examined Material: Brazil, Paraná, Porto Rico, 20.VIII.01, L. C. Rodrigues $w / n^{O}$ (HUEM 16755); 03.VIII.02, L. C. Rodrigues $w / n^{O}$ (HUEM 16758); 02.XI.02, L. C. Rodrigues w/no (HUEM 16759); 08.III.04, L. C. Rodrigues $w / n^{\circ}$ (HUEM 16763); 24.IX.04, L. C. Rodrigues w/no (HUEM 16765); 09.XII.05, S. Jati w/n ${ }^{O}$ (HUEM 16769); 15.IX.06, S. Jati $w / n^{\circ}$ (HUEM 16773); 13.III.09, S. Jati $w / n^{O}$ (HUEM 16782).

Strombomonas fluviatilis (Lemmermann) Deflandre var. levis (Lemmermann) Skvortzov, Archiv für Protistenkunde 69 (3): 551-614, 1930.

Figure 16

Fusiform lorica, circular apical view; smooth wall; anterior pole projected on a curved smooth collar; posterior pole ending in a straight caudal process. Length 65.3- 66.5 $\mu \mathrm{m}$, width 27.0-28.3 $\mu \mathrm{m}$. length caudal process $12.0-13.2 \mu \mathrm{m}$. 
Comment: It is distinguished from the typical variety due to its larger dimensions. The observed specimens constitute a new record for the Upper Paraná River Floodplain.

Occurrence frequency: Sporadic.

Examined Material: Brazil, Paraná, Porto Rico, 02.XI.02, L. C. Rodrigues $w / n^{O}$ (HUEM 16759); 08.III.03, L. C. Rodrigues $w / n^{O}$ (HUEM 16760); 18.VI.04, L. C. Rodrigues $w / n^{\circ}$ (HUEM 16764); 24.IX.04, L. C. Rodrigues $w / n^{O}$ (HUEM 16765); 16.IX.05, S. Jati w/no (HUEM 16768); 10.III.06, S. Jati $w / n^{O}$ (HUEM 16770).

Strombomonas fluviatilis (Lemmermann) Deflandre var. fluviatilis, Archiv für Protistenkunde 69 (3): 580, fig. 52, 53, 1930.

Figure 17

Ellipsoid lorica; circular apical view; smooth wall; short and straight collar; short and curved caudal process. Length 20.9-39.4 $\mu \mathrm{m}$, width 13.0-24.6 $\mu \mathrm{m}$, length collar $2.6 \mu \mathrm{m}$, length caudal process $2.6 \mu \mathrm{m}$

Occurrence frequency: Sporadic.

Examined Material: Brazil, Paraná, Porto Rico, 20.VIII.01, L. C. Rodrigues $w / n^{O}$ (HUEM 16755); 02.XI.02, L. C. Rodrigues $w / n^{O}$ (HUEM 16759); 02.XII.04, L. C. Rodrigues $w / n^{O}$ (HUEM 16762); 24.IX.04, L. C. Rodrigues $w / n^{\circ}$ (HUEM 16765); 03.III.05, S. Jati w/n (HUEM 16766).

Strombomonas triquetra (Playfair) Deflandre var. torta Rino, Revista de Ciências Biológicas, 5: 169, pl. 9, fig. 7-10. 1972.

Figure 18

Rectangular lorica, nearly parallel in the $2 / 3$ anterior and triangular at the distal portion; smooth walls; triangular in apical view, short and large collar; short and conical caudal process, $2.6 \mu \mathrm{m}$. Length $36.5 \mu \mathrm{m}$, width $18.3 \mu \mathrm{m}$, length collar $2.6 \mu \mathrm{m}$.

Comment: The observed specimens constitute a new record for the Upper Paraná River Floodplain.

Occurrence frequency: Rare.

Examined Material: Brazil, Paraná, Porto Rico, 27.XI.08, S. Jati $w / n^{O}$ (HUEM 16779).

Trachelomonas acanthophora Stokes var. acanthophora, Proceedings of the American Philosophical Society 33: 340, pl. XXI, fig.6, 1894.

Figure 19

Lageniform lorica; wall ornamented with spines; developed collar decorated with six conical spines; long and cylindrical caudal process with three acute, divergent spines, implanted in its distal pole. Length 39.1-51.4 $\mu \mathrm{m}$, width 18.3-23.1 $\mu \mathrm{m}$, spines $5.2 \mu \mathrm{m}$, length collar $5.2 \mu \mathrm{m}$, length caudal process $7.8 \mu \mathrm{m}$.
Comment: The observed specimens constitute a new record for the Upper Paraná River Floodplain.

Occurrence frequency: Sporadic.

Examined Material: Brazil, Paraná, Porto Rico, 08.III.03, L. C. Rodrigues $w / n^{O}$ (HUEM 16760); 08.III.04, L. C. Rodrigues $w / n^{O}$ (HUEM 16763); 24.IX.04, L. C. Rodrigues $w / n^{O}$ (HUEM 16765); 03.III.05, S. Jati $w / n^{O}$ (HUEM 16766); 13.III.09, S. Jati $w / n^{O}$ (HUEM 16782).

Trachelomonas amphoriformis Osorio-Taffal var. spinosa Conforti, Rea. Hydrobiol. trop. 26 (1): 8, fig. 15, 1993.

Figure 20

Fusiform lorica; wall with granulations distributed irregularly; anterior pole with a tall collar, with granulations; posterior pole ending in an acute caudal process with granulations. Length $64-70.5 \mu \mathrm{m}$, width $12.3-18.3 \mu \mathrm{m}$, length collar 6.1-7.4 $\mu \mathrm{m}$.

Comment: Conforti (1993) acknowledges the presence of spines in the wall. The observed specimens constitute a new record for the Upper Paraná River Floodplain.

Occurrence frequency: Rare.

Examined Material: Brazil, Paraná, Porto Rico, 13.III.09, S. Jati $w / n^{O}$ (HUEM 16782).

Trachelomonas armata (Ehrenberg) Stein var. longispina Playfair emend. Deflandre, Nemours, v. 88, pl. 6, fig. 330. 1926.

Figure 21

Oval-expanded lorica; wall ornamented with short spines distributed all over the lorica and bigger spines at the posterior pole; short collar surrounded by spines. Length 36.9-54.6 $\mu \mathrm{m}$, width 23.1-39.6 $\mu \mathrm{m}$.

Comment: This taxon is different from the typical variety as it presents an oval-expanded lorica and larger length, spines in the anterior pole and considerably larger spines in the posterior pole (Deflandre, 1926, Araujo et al., 2012). The observed specimens constitute a new record for the Upper Paraná River Floodplain.

Occurrence frequency: Frequent.

Examined Material: Brazil, Paraná, Porto Rico, 09.II.01, L. C. Rodrigues w/n $n^{O}$ (HUEM 16753); 20.VIII.01, L. C. Rodrigues $w / n^{O}$ (HUEM 16755); 02.XI.02, L. C. Rodrigues $w / n^{O}$ (HUEM 16759); 08.III.03, L. C. Rodrigues $w / n^{\circ}$ (HUEM 16760); 03.IX.03, L. C. Rodrigues w/no (HUEM 16761); 02.XII.04, L. C. Rodrigues $w / n^{O}$ (HUEM 16762); 08.III.04, L. C. Rodrigues $w / n^{O}$ (HUEM 16763); 18.VI.04, L. C. Rodrigues $w / n^{\circ}$ (HUEM 16764); 24.IX.04, L. C. Rodrigues $w / n^{\circ}$ (HUEM 16765); 
03.III.05, S. Jati w/n $n^{O}$ (HUEM 16766); 10.VI.05, S. Jati $w / n^{O}$ (HUEM 16767); 10.III.06, S. Jati $w / n^{O}$ (HUEM 16770); 23.VI.06, S. Jati w/no (HUEM 16771); 29.II.08, S. Jati w/no (HUEM 16778); 27.XI.08, S. Jati w/n ${ }^{O}$ (HUEM 16779).

Trachelomonas abrupta Swirenko emend. Deflandre var. obesa (Playfair) Deflandre, Nemours, p. 93. fig. 353, 362, 363. 1926.

Figure 22

Ellipsoid to obovoid lorica; strong and irregularly punctate wall, usually scrobiculate; no spines; ring thickening. Length $24.5 \mu \mathrm{m}$, width $19.4 \mu \mathrm{m}$.

Comment: This taxon is different from the typical variety for presenting larger width. The observed specimens constitute a new record for the Upper Paraná River Floodplain.

Occurrence frequency: Rare.

Examined Material: Brazil, Paraná, Porto Rico, 09.II.01, L. C. Rodrigues $w / n^{O}$ (HUEM 16753); 24.IX.04, L. C. Rodrigues w/no (HUEM 16765); 03.III.05, S. Jati w/no (HUEM 16766).

Trachelomonas cupula Deflandre. Revue Générale de Botanique 38: 584, pl.6, figs. 97-99, 1926.

Figure 23

Hemispheric lorica; finely punctuated wall; flattened anterior pole, developed ring thickening; triangular posterior pole. Length $12.3-14.8 \mu \mathrm{m}$, width $15.6-19.9$.

Occurrence frequency: Sporadic.

Examined Material: Brazil, Paraná, Porto Rico, 08.III.04, L. C. Rodrigues w/no (HUEM 16763); 18.VI.04, L. C. Rodrigues w/no (HUEM 16764); 10.VI.05, S. Jati w/no (HUEM 16767); 29.II.08, S. Jati $w / n^{\circ}$ (HUEM 16778).

Trachelomonas cervicula Stokes var. cervicula, Proceedings of the American Philosophical Society 28:75, fig 11. 1890.

Figure 24

Spherical lorica; smooth wall; presence of ring thickening with cylindrical collar, inclined or not, oriented to the inside of the cell. Diameter 15.3-17.2 $\mu \mathrm{m}$, length collar 7.4 $\mu \mathrm{m}$.

Occurrence frequency: Sporadic.

Examined Material: Brazil, Paraná, Porto Rico, 11.V.00, L. C. Rodrigues $w / n^{O}$ (HUEM 16754); 02.XII.04, L. C. Rodrigues $w / n^{O}$ (HUEM 16762); 08.III.04, L. C. Rodrigues $w / n^{\circ}$ (HUEM 16763); 24.IX.04, L. C. Rodrigues w/no (HUEM 16765); 10.III.06, S. Jati w/no (HUEM 1770); 13.III.09, S. Jati $w / n^{O}$ (HUEM 16782).

Trachelomonas decora Deflandre var. decora, Monographie du genre Trachelomonas Ehr. 121, Pl.11, fig. 631. 1926.
Figure 25a and 25b

Elongated-cylindrical ellipsoid lorica; wall densely ornamented with short spines except in the area near the collar and caudal process; infundibuliform short collar, conical, cast spines, short at the distal edge forming a crown; sub-cylindrical caudal process, long and with spines only at the distal pole. Length $39.8 \mu \mathrm{m}$, width $18.4 \mu \mathrm{m}$.

Comment: Trachelomonas decora Deflandre var. decora constitutes the first citation of occurrence for the Upper Paraná River Floodplain.

Occurrence frequency: Rare.

Examined Material: Brazil, Paraná, Porto Rico, 24.IX.04, L. C. Rodrigues $w / n^{O}$ (HUEM 16765).

Trachelomonas hispida (Perty) Stein emend. Deflandre var. coronata Lemmermann, in Pascher, Süsswasserfl. Deutsch. 2 (2): 150. 1913.

Figure 26

Ellipsoid lorica; sides nearly parallel; punctuated wall ornamented with spines distributed all over the lorica; sharp ring thickening covered with a crown of spines. Length 22.9-31.5 $\mu \mathrm{m}$, width 18.7-27 $\mu \mathrm{m}$.

Comment: Differs from the typical variety for presenting collar covered with spines identical to those in the lorica. The observed specimens constitute a new record for the Upper Paraná River Floodplain.

Occurrence frequency: Frequent.

Examined Material: Brazil, Paraná, Porto Rico, 11.V.00, L. C. Rodrigues w/no (HUEM 16754); 03.VIII.02, L. C. Rodrigues $w / n^{O}$ (HUEM 16758); 02.XI.02, L. C. Rodrigues w/no (HUEM 16759); 08.III.03, L. C. Rodrigues $w / n^{\circ}$ (HUEM 16760); 03.IX.03, L. C. Rodrigues $w / n^{O}$ (HUEM 16761); 02.XII.04, L. C. Rodrigues $w / n^{O}$ (HUEM 16762); 24.IX.04, L. C. Rodrigues w/no (HUEM 16765); 03.III.05, S. Jati w/no (HUEM 16766); 09.XII.05, S. Jati $w / n^{O}$ (HUEM 16769); 10.III.06, S. Jati $w / n^{O}$ (HUEM 16770); 23.VI.06, S. Jati $w / n^{O}$ (HUEM 16771).

Trachelomonas megalacantha Cunha var. megalacantha, Memórias do Instituto Oswaldo Cruz 6(3): 172, fig. 5. 1914.

Figure 27

Elliptic-elongated loric; wall punctuated and ornamented with long spines, distributed irregularly throughout the cell; flagellar pore with finite annular formed by the fusion of short conical spines. Length $46.9 \mu \mathrm{m}$, width $31.6 \mu \mathrm{m}$, length spines $11.2 \mu \mathrm{m}$.

Comment: The observed specimens constitute a new record for the Upper Paraná River Floodplain. 
Occurrence frequency: Rare.

Examined Material: Brazil, Paraná, Porto Rico, 10.III.06, S. Jati w/no (HUEM 16770).

Trachelomonas molesta Deflandre var. molesta Revie Algologique 3 (1-2): 223, fig. 74, 1928.

Figure 28

Fusiform lorica; densely punctuated wall; cylindrical tall collar with serrated distal pole, external ring thickening; hollow caudal process with a cross-section membrane separating it from the remaining of the lorica. Length $67.9 \mu \mathrm{m}$, width $20.9 \mu \mathrm{m}$, collar $7.8 \mu \mathrm{m}$.

Comment: Trachelomonas molesta and Trachelomonas allorgei are very similar and differ only regarding the presence of a membrane separating the caudal process from the remaining of the lorica in Trachelomonas molesta. For Tell \& Conforti (1986), both taxa are independent. Menezes \& Fernandes (1989) assumed Trachelomonas allorgei var. molesta. Dillard (2000), kept both taxa separated and referred to the caudal process of Trachelomonas molesta as a thorn. We agree with Tell \& Conforti (1986) and Dillard (2000) and identified the observed specimens as Trachelomonas molesta Deflandre. The specimens constitute a new record for the Upper Paraná River Floodplain.

Occurrence frequency: Rare.

Examined Material: Brazil, Paraná, Porto Rico, 08.III.03, L. C. Rodrigues $w / n^{O}$ (HUEM 16760).

Trachelomonas obtusa Palmer var. obtusa, Proceedings of the Academy of Natural Sciences of Philadelphia, 57: 673, pl. 41, fig. 3, 1905.

Figure 29

Cylindrical-conical lorica, nearly parallel sides; wall thickly covered by short conical spines; flagellar pore with short collar or without it; apical pole slightly convex; tapered posterior pole with slightly rounded sides. Length 19.7-23.5 $\mu \mathrm{m}$, width 12.2- $15.7 \mu \mathrm{m}$, collar $2.6 \mu \mathrm{m}$.

Comment: The observed specimens constitute a new record for the Upper Paraná River Floodplain.

Occurrence frequency: Rare.

Examined Material: Brazil, Paraná, Porto Rico, 16.IX.05, S. Jati w/n ${ }^{\circ}$ (HUEM 16768); 13.III.09, S. Jati $w / n^{O}$ (HUEM 16782).

Trachelomonas parvicollis Deflandre var. parvicollis, Révue Générale de Botanique 38: 704. pl. 9, fig. 493, 1927.

Figure 30

Subspherical lorica; smooth wall; pore with a long, cylindrical collar projected towards the outside of the lorica; rounded anterior and posterior poles.
Length 18.9-25.5 $\mu \mathrm{m}$, width 17.8-24 $\mu \mathrm{m}$, collar 4.2- $6 \mu \mathrm{m}$.

Comment: Tell \& Conforti (1986) found in the specimens studied a cellular wall finely punctuated and with much spacing. However, the specimens found at Pau Velho Backwater, as well as those observed by Deflandre (1926), present smooth cellular wall.

Occurrence frequency: Sporadic.

Examined Material: Brazil, Paraná, Porto Rico, 11.V.00, L. C. Rodrigues $w / n^{O}$ (HUEM 16754); 08.III.03, L. C. Rodrigues $w / n^{\circ}$ (HUEM 16760); 02.XII.04, L. C. Rodrigues $w / n^{O}$ (HUEM 16762); 18.VI.04, L. C. Rodrigues $w / n^{O}$ (HUEM 16764); 24.IX.04, S. Jati w/n ${ }^{O}$ (HUEM 16765); 03.III.06, S. Jati $w / n^{O}$ (HUEM); 10.III.06, S. Jati $w / n^{O}$ (HUEM 16770); 01.XII.06, S. Jati w/no (HUEM 16772); 13.III.09, S. Jati w/n ${ }^{O}$ (HUEM 16782).

Trachelomonas rugulosa Stein emend. Deflandre. var. rugulosa fa. paralela Tell \& Zalocar De Domitrovic, Nova Hedigia, 41: 353-391, 1985.

Figure 31

Subspherical lorica; wall ornamented with prominent striae, longitudinally slightly anastomosed; short collar. Length 15.7-26.4 $\mu \mathrm{m}$, width 14.7-23.1 $\mu \mathrm{m}$.

Occurrence frequency: Frequent.

Examined Material: Brazil, Paraná, Porto Rico, 28.II.02, L. C. Rodrigues $w / n^{O}$ (HUEM 16756); 05.V.02, L. C. Rodrigues $w / n^{O}$ (HUEM 16758); 02.XI.02, L. C. Rodrigues $w / n^{\circ}$ (HUEM 16759); 08.III.03, L. C. Rodrigues w/no (HUEM 16760); 02.XII.04, L. C. Rodrigues $w / n^{O}$ (HUEM 16762); 08.III.04, L. C. Rodrigues w/no (HUEM 16763); 03.III.05, S. Jati w/no (HUEM 16766); 10.VI.05, S. Jati $w / n^{\circ}$ (HUEM 16767); 09.XII.05, S. Jati $w / n^{O}$ (HUEM 16769); 10.III.06, S. Jati $w / n^{O}$ (HUEM 16770).

Trachelomonas sculpta Balech var. sculpta, Analess del Museo Argentino de Ciencias Naturales "Bernardino Rivadavia", 41: 245, fig. 32, fig. 167. 1944.

Figure 32

Spherical lorica; wall ornamented with depressions approximately polygonal, isometric and regulaly distributed, distributed all over the lorica, without spines; short collar. Diameter 18.3-20.9 $\mu \mathrm{m}$.

Occurrence frequency: Frequent.

Examined Material: Brazil, Paraná, Porto Rico, 11.V.00, L. C. Rodrigues $w / n^{O}$ (HUEM 16754); 09.II.01, L. C. Rodrigues $w / n^{O}$ (HUEM 16753); 28.II.02, L. C. Rodrigues $w / n^{O}$ (HUEM 16756); 05.V.02, L. C. Rodrigues w/no (HUEM 16758); 
02.XI.02, L. C. Rodrigues $w / n^{O}$ (HUEM 16759); 08.III.03, L. C. Rodrigues w/no (HUEM 16760); 03.IX.03, L. C. Rodrigues $w / n^{O}$ (HUEM 16761); 02.XII.04, L. C. Rodrigues $w / n^{O}$ (HUEM 16762); 08.III.04, L. C. Rodrigues w/no (HUEM 16763); 18.VI.04, L. C. Rodrigues w/no (HUEM 16764); 24.IX.04, L. C. Rodrigues w/no (HUEM 16765); 03.III.05, S. Jati w/no (HUEM 16766); 10.VI.05, S. Jati $w / n^{O}$ (HUEM 16767); 16.IX.05, S. Jati $w / n^{\circ}$ (HUEM 16768); 09.XII.05, S. Jati w/n ${ }^{\circ}$ (HUEM 16769), 15.IX.06, S. Jati $w / n^{O}$ (HUEM 16773), 29.II.08, S. Jati $w / n^{O}$ (HUEM 16778).

Trachelomonas similis Stokes var. similis, Proceedings of the American Philosophical Society, 28:76, fig. 12. 1890.

Figure 33

Ellipsoidal lorica; wall ornamented with punctuations; curved collar. Length $20.4 \mu \mathrm{m}$, width $16.3 \mu \mathrm{m}$, diameter collar $3.1 \mu \mathrm{m}$.

Comment: The specimens analyzed constitute a new record for the Upper Paraná River Floodplain.

Occurrence frequency: Sporadic.

Examined Material: Brazil, Paraná, Porto Rico, 11.V.00, L. C. Rodrigues $w / n^{O}$ (HUEM 16754); 09.II.01, L. C. Rodrigues $w / n^{O}$ (HUEM 16753); 28.II.02, L. C. Rodrigues $w / n^{O}$ (HUEM 16756); 05.V.02, L. C. Rodrigues $w / n^{O}$ (HUEM 16758); 02.XII.04, L. C. Rodrigues $w / n^{O}$ (HUEM 16762); 24.IX.04, L. C. Rodrigues w/no (HUEM 16765); 09.XII.05, S. Jati w/n ${ }^{O}$ (HUEM 16769); 23.VI.06, S. Jati $w / n^{O}$ (HUEM 16771); 01.XII.06, S. Jati $w / n^{O}$ (HUEM 16772); 27.XI.08, S. Jati w/n (HUEM 16779); 13.III.09, S. Jati w/no (HUEM 16782).

Trachelomonas similis Stokes var. spinosa Huber-Pestalozzi, Phytoplankton des Süsswasser 16 (4): 342, pl. 73, fig. 722a, 1955.

Figure 34

Distinguished from the typical variety for having the wall ornamented with spines distributed all over the lorica and in the curved collar. Length 23.5-29.7 $\mu \mathrm{m}$, width 18.3-23.1 $\mu \mathrm{m}$, spines, $6.6 \mu \mathrm{m}$, collar diameter 2.6-4.2 $\mu \mathrm{m}$.

Occurrence frequency: Rare.

Examined Material: Brazil, Paraná, Porto Rico, 13.III.09, S. Jati w/n $($ HUEM 16782).

Trachelomonas volvocina (Ehrenberg) Ehernberg var. volvocina, Abhandlungen der königlichen Akademie der wissenschaften zu Berlin 1833:315, pl. 7, fig. 3, 1835.

Figure 35
Spherical lorica; smooth wall; short collar; two lateral chloroplasts with double pyrenoids. Diameter 17.2-19.7 $\mu \mathrm{m}$.

Comment: The safe differentiation between T. volvocinopsis and T. volvocina is determined by observing the amount of chloroplasts, which are numerous and present no pyrenoids at the first and between two or three laterals with double pyrenoids at the second. The observed specimens constitute a new record for the Upper Paraná River Floodplain.

Occurrence frequency: Rare.

Examined Material: Brazil, Paraná, Porto Rico, 27.XI.08, S. Jati w/no (HUEM 16779); 13.III.09, S. Jati $w / n^{O}$ (HUEM 16782).

Trachelomonas volvocinopsis Swirenko var. volvocinopsis, Archiv für Hydrobiologie und Planktonkunde 9: 633, pl. 19: fig. 1-2, 1914.

Figure 36

Spherical lorica; smooth wall; short collar; numerous discoid chloroplasts distributed all over the cytoplasm. Diameter 12.3-29.5 $\mu \mathrm{m}$.

Occurrence frequency: Frequent.

Examined Material: Brazil, Paraná, Porto Rico, 11.V.00, L. C. Rodrigues $w / n^{O}$ (HUEM 16754); 09.II.01, L. C. Rodrigues $w / n^{O}$ (HUEM 16753); 20.VIII.01, L. C. Rodrigues $w / n^{O}$ (HUEM 16755); 28.II.02, L. C. Rodrigues $w / n^{O}$ (HUEM 16756); 05.V.02, L. C. Rodrigues $w / n^{O}$ (HUEM 16758); 03.VIII.02, L. C. Rodrigues $w / n^{O}$ (HUEM 16758); 08.III.03, L. C. Rodrigues $w / n^{\circ}$ (HUEM 16760); 03.IX.03, L. C. Rodrigues $w / n^{O}$ (HUEM 16761); 02.XII.04, L. C. Rodrigues $w / n^{O}$ (HUEM 16762); 08.III.04, L. C. Rodrigues w/no (HUEM 16763); 18.VI.04, L. C. Rodrigues $w / n^{\circ}$ (HUEM 16764); 24.IX.04, L. C. Rodrigues w/no (HUEM 16765); 03.III.05, S. Jati w/no (HUEM 16766); 10.VI.05, S. Jati $w / n^{O}$ (HUEM 16767); 16.IX.05, S. Jati $w / n^{O}$ (HUEM 16768); 09.XII.05, S. Jati $w / n^{O}$ (HUEM 16769); 10.III.06, S. Jati w/no (HUEM 16770); 23.VI.06, S. Jati w/n ${ }^{O}$ (HUEM 16771); 01.XII.06, S. Jati w/n ${ }^{O}$ (HUEM 16772); 15.IX.06, S. Jati $w / n^{O}$ (HUEM 16773); 29.II.08, S. Jati $w / n^{O}$ (HUEM 16778); 27.XI.08, S. Jati w/no (HUEM 16779); 13.III.09, S. Jati w/no (HUEM 16782).

Trachelomonas woycickii Koczwara var. woycickii, Kosmos 40: 231-275. 1915.

Figure 37

Spherical lorica; wall ornamented with short, conical spines distributed all over the lorica; short collar. Diameter 16.5-22.0 $\mu \mathrm{m}$.

Occurrence frequency: Sporadic.

Examined Material: Brazil, Paraná, Porto Rico, 24.IX.2004, L. C. Rodrigues $w / n^{O}$ (HUEM 
16765); 10.VI.2005, S. Jati $w / n^{O}$ (HUEM 16767); 16.IX.2005, S. Jati $w / n^{O}$ (HUEM 16768); 10.III.2006, S. Jati $w / n^{O}$ (HUEM 16770); 29.II.2008, S. Jati w/no (HUEM 16778); 27.XI.2008, S. Jati $w / n^{O}$ (HUEM 779).

Trachelomonas woycickii Koczwara var. pusilla Drezepolski, Kosmos, Warszawa, 50(1A): 265, fig. 15, 1925.

Figure 38

Distinguished from the typical variety for presenting smaller dimensions. Diameter 11.2-12.2 $\mu \mathrm{m}$.

Comment: The material analyzed by Jati \& Train (1994) for temporary lakes of Porto Rico Island revealed only organisms with small dimensions (7.9-10.5 $\mu \mathrm{m})$. However, the authors decided to maintain them within the typical variety of the species. At Pau Velho Backwater, many organisms were found with dimensions equal to the typical variety $(20,0-29,0 \mu \mathrm{m})$ and other much smaller ones (11.2-12.2 $\mu \mathrm{m})$, categorized within the variety pusilla, which differs from the typical variety only due to their smaller cellular dimensions.

Comment: The observed specimens constitute a new record for the Upper Paraná River Floodplain.

Occurrence frequency: Rare.

Examined Material: Brazil, Paraná, Porto Rico, 13.III.09, S. Jati w/no (HUEM 16782).

\section{Conclusion}

This study has enabled the update of the nomenclature of Euglenophyceae registered in these habitats. Among the six (6) taxa described here as belonging to the genera Leponcinclis, three (3) were previously registered as Euglena Ehrenberg and one (1) as Phacus Dujardin. In studies of taxonomic review, Marin et al. (2003) and Kosmala et al. (2005) have transfered Euglena acus Ehrenberg, E. oxyuris Schmarda and E. spirogyra Ehrenberg to the genus Lepocinclis. Bennett \& Triemer (2012), on the other hand, transferred Phacus horridus to Lepocinclis spinosa.

This study has increased the knowledge on the geographic distribution of pigmented Euglenophyceae in the upper Paraná River floodplain and in Brazil: Lepocinclis fusca, Monomorphina pyrum, Phacus contortus, P. longicauda var. major, P. rodriguesiae, Strombomonas fluviatilis var. levis, S. triquetra, Trachelomonas abrupta var. obesa, T. acanthophora, T. amphoriformis var. spinosa, T. armata var. longispina, T. decora, T. hispida var. coronata, T. megalacantha, T. molesta, T. obtusa, T. similis var. similis, T. volvocina var. volvocina and
T. woycickii var. pusilla consisted new occurrence records.

Furthermore, the high occurrence of taxa classified as sporadic and rare $(67 \%)$ is probably related to the high environmental variability observed during the study period.

\section{Acknowledgements}

We thank the Long Term Ecological Research Program (Programa de Pesquisa Ecológica de Longa Duração, PELD/CNPq), and the Center of Research in Limnology, Ichthyology, and Aquaculture of State University of Maringá (Nupélia/UEM) for logistical and financial support. We also thank MSc. Geovani Arnhold Moresco for auxiliating in the elaboration of the figures, and the Laboratory of Basic Limnology/Nupelia for providing abiotic data.

\section{References}

ALVES-DA-SILVA, S.M. and BICUDO, C.E.M. Cryptoglena, Monomorphina and Phacus (Euglenophyceae) of a reservoir in the State of Rio Grande do Sul, southern Brazil. Brazilian Journal of Botany, 2009, 32(2), 253-270. http://dx.doi. org/10.1590/S0100-84042009000200006.

ALVES-DA-SILVA, S.M. and BRIDI, F.C. Estudo de Euglenophyta no Parque Estadual Delta do Jacuí, Rio Grande do Sul, Brasil. 2. Os gêneros Phacus Dujardin e Hyalophacus (Pringsheim) Pochmann. Iheringia. Série Botânica, 2004, 59(1), 75-96.

ALVES-DA-SILVA, S.M. and KLEIN, I.C. Euglenophyceae na Área de Proteção Ambiental do Rio Ibirapuitã, sudoeste do Estado do Rio Grande do Sul, Brasil. 1. Cryptoglena Marin \& Melkonian emend. Kosmala \& Zakrýs, Monomorphina (Ehrenberg) Mereschkowsky emend. Kosmala \& Zackýs e Phacus Durjardin. Hoehnea, 2015, 42(3), 471-496. http:// dx.doi.org/10.1590/2236-8906-12/2015.

ALVES-DA-SILVA, S.M., CABREIRA, J.C., VOOS, J.G. and LOBO, E.A. Species richness of the genera Trachelomonas and Strombomonas (pigmented Euglenophyceae) in a subtropical urban lake in the Porto Alegre Botanical Garden, RS, Brazil. Acta Botanica Brasílica, 2013, 27(3), 526-536. http:// dx.doi.org/10.1590/S0102-33062013000300010.

ALVES-DA-SILVA, S.M., ESCOBAR, K.E. and JULIANO, V.B. Novos registros de Trachelomonas Ehr. emend. Deflandre (Euglenophyceae) para o Estado do Rio Grande do Sul e Brasil. Hoehnea, 2016, 43(1), 1-10. http://dx.doi.org/10.1590/22368906-64/2014.

ALVES-DA-SILVA, S.M., FERRAZ, G.C. and TORRES, J.R. Euglenaceae pigmentadas de dois arroios e do rio Jacuí, regiāo carbonífera do município 
de São Jerônimo, Rio Grande do Sul, Brasil. Revista Brasileira de Biologia, 1991, 51(4), 813-828.

ARAUJO, G.J.M. and BICUDO, C.E.M. Euglenophyceae de águas continentais do Estado de São Paulo: gênero Lepocinclis Perty emend. Marin \& Melkonian in Marin et al. Hoehnea, 2017, 44(2), 295-314. http://dx.doi.org/10.1590/2236-8906$56 / 2016$.

ARAUJO, G.J.M., BARBOSA, J.E.L. and BARBOSA, L.G. Pigmented Euglenophytes in a natural and shallow lake in the semiarid region of Paraíba State, Brazil. Brazilian Journal of Botany, 2012, 35(1), 17-30. http://dx.doi.org/10.1590/S180699592012000100004

BENNETT, M.S. and TRIEMER, R.E. A New Method For Obtaining Nuclear Gene Sequences From Field Samples And Taxonomic Revisions Of The Photosynthetic Euglenoids Lepocinclis (Euglena) helicoideus and Lepocinclis (Phacus) horridus (Euglenophyta). Journal of Phycology, 2012, 48(1), 254-260. http://dx.doi.org/10.1111/j.15298817.2011.01101.x. PMid:27009671.

BICUDO, C.E.M. and MENEZES, M. Gêneros de Algas de Águas Continentais do Brasil: chave para identificação e descriçôes. Sao Carlos: RiMa, 2006.

BICUDO, C.E.M. and MENEZES, M. Phylogeny and Classificaton of Euglenophyceae: A Brief Review. Frontiers in Ecology and Evolution, 2016, 4, 17. http:// dx.doi.org/10.3389/fevo.2016.00017.

BIOLO, S. and RODRIGUES, L. New records of Xanthophyceae and Euglenophyceae in the periphitic algal community from a neotropical river floodplain, Brazil. Algological Studies, 2010, 135(1), 61-81. http://dx.doi.org/10.1127/1864-1318/2010/01350061.

BORGES, P.A.F. and TRAIN, S. Phytoplankton diversity in the Upper Paraná River floodplain during two years of drought (2000 and 2001). Brazilian Journal of Biology = Revista Brasileira de Biologia, 2009, 69(2), 637-647, Supplement. http://dx.doi.org/10.1590/ S1519-69842009000300018. PMid:19738970.

BOVO-SCOMPARIN, V.M., BORGES, P.A.F., TRAIN, S. and RODRIGUES, L.C. Xanthophyceae planctônicas da Upper Paraná River Floodplain. Acta Scientiarum. Biological Sciences, 2005, 27(1), 9-20.

CONFORTI, V. Study of the euglenophyta from Camaleão Lake (Manaus-Brasil). I. Trachelomonas Ehr. Revue Hidrobiologique Tropicale, 1993, 26(1), 3-18.

DAJOZ, R. Principios de Ecologia. Porto Alegre: Artmed, 2005.

DEFLANDRE, G. Monographie du genre Trachelomonas Ehr. Nemours: Andre Lesot, 1926. 162 p.

DILLARD, G.E. Freshwater Algae of the Southeastern United States. Part 7. Pigmented Euglenophyceae.
Berlin and Stuttgart: J. Cramer, 2000, 135 p. Bibliotheca Phycologica, vol. 106.

GINÉ, M.F., BERGAMIN F, H., ZAGATTO, E.A.G. and REIS, B.F. Simultaneous determination of nitrate and nitrite by flow injection analysis. Analytica Chimica Acta, 1980, 114, 191-197. http://dx.doi. org/10.1016/S0003-2670(01)84290-2.

GOLTERMAN, H.L., CLYMO, R.S. and OHNSTAD, M.A.M. Methods for physical and chemical analysis of freshwater. 2nd ed. Oxford: IBP, 1978. Blackwell Scientific Publication.

HUBER-PESTALOZZI, G. Euglenophyceen. In: G. Huber-Pestalozzi, ed. Das phytoplankton des Susswässers: Systematik und Biologie. Stuttgart: E. Schweizerbart'sche Verlagsbuchhandlung, 1955. Teil 4.

JATI, S. and TRAIN, S. Euglenophycea pigmentadas de environments lênticos da Ilha Porto Rico, Município de Porto Rico, Paraná, Brazil. Iheringia, 1994, 45, 117-142.

JATI, S. and TRAIN, S. Representantes do gênero Trachelomonas Ehrenberg de duas lagoas da ilha Porto Rico, município de Porto Rico, Paraná, Brasil. Revista Unimar, 1993, 15, 37-51, Supplement.

JATI, S., RODRIGUES, L.C., BORTOLINI, J.C., PAULA, A.C.M., MORESCO, G.A., REIS, L.M., ZANCO, B.F. and TRAIN, S. First record of the occurrence of Ceratium furcoides (Levander) Langhns (Dinophyceae) in the Upper Paraná River Floodplain (PR/MS) Brazil. Brazilian Journal of Biology = Revista Brasileira de Biologia, 2014, 77(3), 235-236, Supplement. http://dx.doi.org/10.1590/15196984.19313.

KIM, J.I., SHIN, W. and TRIEMER, R.E. Phylogenetic Reappraisal of the Genus Monomorphina (Euglenophyceae) Based on Molecular and Morphological Data. Journal of Phycology, 2013, 49(1), 82-91. http://dx.doi.org/10.1111/jpy.12018. PMid:27008391.

KOROLEFF, K. Determination of Ammonia. In: K. GRASSHOFF and E. KREMLING, eds. Methods of seawater analysis. Weinheim: Verlag Chemie, 1978.

KOSMALA, S., KARNKOWSKA, A., MILANOWSKI, R., KWIATOWSKI, J. and ZAKRYS, B. Phylogenetic and taxonomic position of Lepocinclis fusca comb. nov. (= euglena fusca) (euglenaceae): morphological and molecular justification. Journal of Phycology, 2005, 41(6), 1258-1267. http://dx.doi. org/10.1111/j.1529-8817.2005.00141.x.

KUFNER, D.C.L. and GIANI, A. Euglenophyta de lagoas da região da Nhecolândia, Pantanal SulMatogrossense, Brasil. Hoehnea, 2017, 44(2), 277-294. http://dx.doi.org/10.1590/2236-8906$21 / 2017$.

MACKERETH, F.Y.H., HERON, J.R. and TAILING, J.F. Water analysis: some revised methods for limnologists. 
Amblesie: Freshwater Biological Association, Titus Wilson and Sons Ltda, 1978.

MARIN, B., PALM, A., KLINGBERG, M. and MELKONIAN, M. Phylogeny and Taxonomic Revision of PlastidContaining Euglenophytes based on SSU rDNA Sequence Comparions and Synapomorphic Signatures in the SSU rRNA Secondary Structure. Protist, 2003, 154(1), 99-145. http://dx.doi.org/10.1078/143446103764928521. PMid:12812373.

MENEZES, M. and FERNANDES, V.O. Euglenaceae (Euglenophyceae) Pigmentadas do Noroeste do Estado de Mato Grosso, Brasil: Município de Barra do Bugres, Cáceres, Juína e Porto Esperidião. Hoehnea, 1989, 16, 35-55.

MENEZES, M. Polimorfismo em Lepocinclis ovum (Ehrenberg) Lemmermann e suas implicaçóes taxonômicas. Rickia, 1987, 14, 1-6.

MORESCO, G.A., PAULA, A.C.M., BORTOLINI, J.C., JATI, S., REIS, L.M. and RODRIGUES, L.C. Zygnemaphyceae em um lago de várzea na Upper Paraná River Floodplain: Genus Closterium, Cosmarium, Euastrum, Micrasterias e Pleurotaenium. Iheringia. Série Botânica, 2015, 70, 143-155.

NEIFF, J.J. Ideas para la interpretacion ecologica del Paraná. Interciencia, 1990, 15(6), 424-441.

OLIVEIRA, M.D., JATI, S. and TRAIN, S. Levantamento preliminar de fitoplancton de rede (exceto zignemaphyceae) do rio Paraná, no município de Porto Rico, Paraná, Brazil. Unimar, 1994, 16, 155-174, Suplemento 3.
PAULA, A.C.M., MORESCO, G.A., BORTOLINI, J.C., JATI, S., REIS, L.M. and RODRIGUES, L.C. Os genus Staurastrum, Staurodesmus e Xanthidium em uma lake de inundação, Upper Paraná River Floodplain, Brazil. Iheringia. Série Botânica, 2014, 69, 417-431.

SOUZA FILHO, E.E. Evaluation of the Upper Paraná River discharge controlled by reservoirs. Brazilian Journal of Biology $=$ Revista Brasileira de Biologia, 2009, 69(2), 707-716, Supplement. http:// dx.doi.org/10.1590/S1519-69842009000300024. PMid:19738976.

TELL, G. and CONFORTI, V. Euglenophyta pigmentadas de la Argentina. Bibliotheca Phycologica, Berlin, Stuttgart. J. Cramer, 1986, 75, 301.

THOMAZ, S.M., PAGIORO, T.A., BINI, L.M., ROBERTO, M.C. and ROCHA, R.R.A., Limnological characterization of the aquatic environments and the influence of hydrometric levels. In: S.M. THOMAZ, A.A. AGOSTINHO and N.S. HAHN, eds. The Upper Paraná River floodplain: physical aspects, ecology and conservatiuon. Leiden: Backhuys Publishers, 2004, pp. 75-102.

TRAIN, S. and RODRIGUES, L.C. Phytoplankton assemblages. In: S.M. THOMAZ, A.A. AGOSTINHO and N.S. HAHN, eds. The Upper Paraná river floodplain: physical aspects, ecology and conservation. Leiden: Backhuys Publishers, 2004, pp. 103-124.

Received: 23 June 2017 Accepted: 03 August 2018 